\title{
Nanoindentation and nanoscratch behaviors of DLC coatings on different steel substrates
}

\author{
T.H. Zhang *, Y. Huan \\ State Key Laboratory of Nonlinear Mechanics (LNM), Institute of Mechanics, Chinese Academy of Sciences, Beisihuan West Road 15, \\ Beijing 100080, China
}

Available online 19 January 2005

\begin{abstract}
Nanoindentation and nanoscratch tests were performed for diamond-like carbon (DLC) coatings on the different steel substrates in order to investigate the deformation and failure behaviors of the coating/substrate systems and their tribological properties. In this work, DLC coatings with a thickness of approximate $500 \mathrm{~nm}$ were grown on 9Cr18 and 40CrNiMo steel substrates by vacuum magnetic-filtering arc plasma deposition, respectively. The nanoindentation results show that the indentation response was plasticity-dominated. The peak load on sample, residual indentation depth, hardness, modulus can provide important information of the mechanical resistance of the materials tested. The scratch process with the ramping normal load was analyzed into the three regimes, which are fully elastic recovery, plastic deformation and delamination of coatings. This shows that the scratch response was controlled by plastic deformation in the substrate. The substrate plays an important role in determining the mechanical properties and wear resistance of such coatings. As a consequence, $9 \mathrm{Cr} 18$ steel is a better candidate of substrate materials for DLC coatings due to the better load-carrying capacity and scratch/wear resistance of DLC/9Cr18.
\end{abstract}

(C) 2005 Elsevier Ltd. All rights reserved.

Keywords: A. Coating; B. Mechanical properties

\section{Introduction}

The diamond-like carbon (DLC) coatings have been developed for a wide range of technological and industrial applications. It is a tribological coating used mainly in the field of mechanical engineering in order to reduce friction and wear, such as drilling and cutting tools. It is also interesting with respect to various applications in the hard disk as well as in the microelectromechanical system (MEMS). The typical coating thickness for these application ranges from some nanometers up to a few hundred nanometers and the applied substrates cover a broad range of materials [1,2]. Therefore, it is essential to search for some methods to appropriately evaluate \footnotetext{
9511.

${ }^{*}$ Corresponding author. Tel.: +86 106254 1733; fax: +86 106257

E-mail address: zhangth@Inm.imech.ac.cn (T.H. Zhang).
}

the mechanical and tribological behavior of DLC coatings.

Staedler and Schiffmann [2] have systematically investigated the correlation of nanomechanical and nanotribological behaviors of thin DLC coatings on different substrates (AlTiC, Si(100), fused silica, SU8 photoresist) using nanoindentation and nanoscratch techniques. The substrates cover the range of very soft (SU8 photoresist) up to very hard (AlTiC ceramic) materials showing a clear influence on the tribological response of the DLC/substrates. Therefore, the primary focus of the research is to investigate the mechanical and tribological properties of DLC coatings on different steel substrates in order to gain a better understanding of the predominant deformation mechanisms that occurred in response to nanoindentation and nanoscratch and further choosing the ideal steel substrate. 


\section{Experimental details}

The substrate materials were: $9 \mathrm{Cr} 18$ bearing steel (Si-0.8, Mn-0.72, P-0.035, S-0.03, C-0.96, Cr-17.8, and $\mathrm{Fe}-79.655)$; and 40CrNiMo alloy structure steel (Si0.25 , Mn-0.70, C-0.41, Cr-0.82, Ni-1.45, Mo-0.18, and Fe-96.19). The specimens were cut from real bearing rings that had been quenched and tempered. Before deposition, the specimens were first mechanically ground and progressively polished, then cleaned in acetone by an ultrasonic washer. The DLC coatings with the thickness of about $500 \mathrm{~nm}$ were prepared utilizing a vacuum magnetic-filtering arc plasma system. The interface adhesion between the coating and the substrate was controlled by a Ti bombardment, i.e., the specimen without the bombardment is nominal with poor adhesion. Four batches of samples were prepared and denoted as $9 \mathrm{Cr} 18,40 \mathrm{CrNiMo}, \mathrm{DLC} / 9 \mathrm{Cr} 18$ and DLC/40CrNiMo. After performing relative nanoindentation and nanoscratch tests, the DJB823 organic coatings were deposited on the DLC/9Cr18 and DLC/ 40CrNiMo samples and denoted as organic/DLC/ $9 \mathrm{Cr} 18$ and organic/DLC/40CrNiMo.

Nanoindentation tests were conducted using a MTS Nano Indenter ${ }^{\circledR}$ XP with a Berkovich diamond tip. Hardness and elastic modulus were measured using the continuous stiffness measurement (CSM) option. A fused silica was used as a standard sample for the initial calibration. In the present study, a typical indentation experiment consists of seven subsequent segments: approaching the surface; determining the contact point; loading to the peak load; holding the tip for $10 \mathrm{~s}$ at the peak load; unloading $90 \%$ of peak load; holding the tip for $100 \mathrm{~s}$ at $10 \%$ of the peak load for thermal drift correction; and finally unloading completely. The experimental parameters were chosen as follows: strain rate, $0.05 \mathrm{~s}^{-1}$; allowable drift rate, $0.05 \mathrm{~nm} / \mathrm{s}$; depth limit, $1.0 \mu \mathrm{m}$. The hardness and elastic modulus were obtained from the curves using Oliver-Pharr method [3]. Nanoscratch tests were performed by a Berkovich tip with face forward using the LFM (Lateral Force Measurement) option of the Nano Indenter ${ }^{\circledR}$ XP. During a scratch test, the ramping normal load on the indenter was held. For these tests, the maximum normal loads were 40 and $300 \mathrm{mN}$, respectively. The scratch velocity was kept at $10 \mu \mathrm{m} / \mathrm{s}$ by controlling the $\mathrm{X}-\mathrm{Y}$ stage movement. A typical scratch experiment is performed in nine subsequent segments: approaching the surface; first profile with normal load $20 \mu \mathrm{N}$ for $700 \mu \mathrm{m}$ in $\mathrm{Y}$ direction; return profile; pre-profile for $100 \mu \mathrm{m}$; scratch profile with ramping normal load for $500 \mu \mathrm{m}$; unload; postprofile for $100 \mu \mathrm{m}$; return profile; final profile. Five indent or scratch tests were performed for each sample. All tests were carried out at $23^{\circ} \mathrm{C}$. Surface observation was performed under a POLYVAR MET $^{\circledR}$ optical microscope.

\section{Results and discussion}

\subsection{Nanoindentation properties}

Nanoindentation tests with a maximum depth of $1000 \mathrm{~nm}$ were performed on four samples: $40 \mathrm{CrNiMo}$, 9Cr18, DLC/40CrNiMo, and DLC/9cr18, respectively. Figs. 1(a)-(c) show the load on sample, hardness and modulus values as a function of indentation depth. Each curve represents the averaged result of five separate indents, and the error bars were calculated from the standard deviation of these five indents. The error values could be correlated with the non-homogeneous component distribution in the samples. The peak load on sample, $P_{\mathrm{m}}$, and hardness exhibit the load-carrying capacity. $1-\delta_{\mathrm{r}}\left(\delta_{\mathrm{r}}\right.$ represents the ratio of the residual depth over the maximum depth) and modulus represent the indentation recovery capacity. The high values of $P_{\mathrm{m}}, 1-\delta_{\mathrm{r}}$, hardness, and modulus can provide us one of standards on how to select the best substrate for DLC coatings. By Comparison with the four parameters between $9 \mathrm{Cr} 18$ and $40 \mathrm{CrNiMo}$, one concludes that $9 \mathrm{Cr} 18$ is a better candidate of substrate material for DLC coatings.

Note the hardness in the topmost $20 \mathrm{~nm}$ of Fig. 1(b). This is likely an artifact of the data reduction method - when the penetration depth is sufficiently shallow, the load-depth curve shows almost complete elastic recovery [4]. Variations in surface topography also led to the scatter in measured values within $50 \mathrm{~nm}$ of the coating surface [5]. The hardness and modulus values of $40 \mathrm{CrNiMo}$ and $9 \mathrm{Cr} 18$ were approximately independent of indentation depths. On the contrary, those of DLC/40CrNiMo and DLC/9Cr18 were dependent on indentation depths. In the two coatings, all data show that maximum hardness and modulus are about 65 and $650 \mathrm{GPa}$ over the depth range from 50 to $100 \mathrm{~nm}$, which is consistent with the general practice of keeping depth $<20 \%$ of the film thickness to minimize substrate effects [6]. The values should be for DLC coatings. With the depth increasing, the hardness and modulus curves begin to decrease due to substrate effects, and gradually lever off at relative steel substrates. Clearly, it represents a composite property, consisting of a contribution from the DLC coating and the substrate. The contribution of the underlying softer substrate became more pronounced with a higher indentation depth. Compared to DLC/40CrNiMo, the hardness and modulus curves for DLC/ $9 \mathrm{Cr} 18$ declined slowly with further increasing indentation depth. The internal stress field can be reduced through improving adhesion properties of coating and substrate.

Fig. 1(d) shows the typical load-depth curves of DLC/9Cr18 and DLC/40CrNiMo. Some discontinuous steps are observed in the two curves. In fact, the steps 

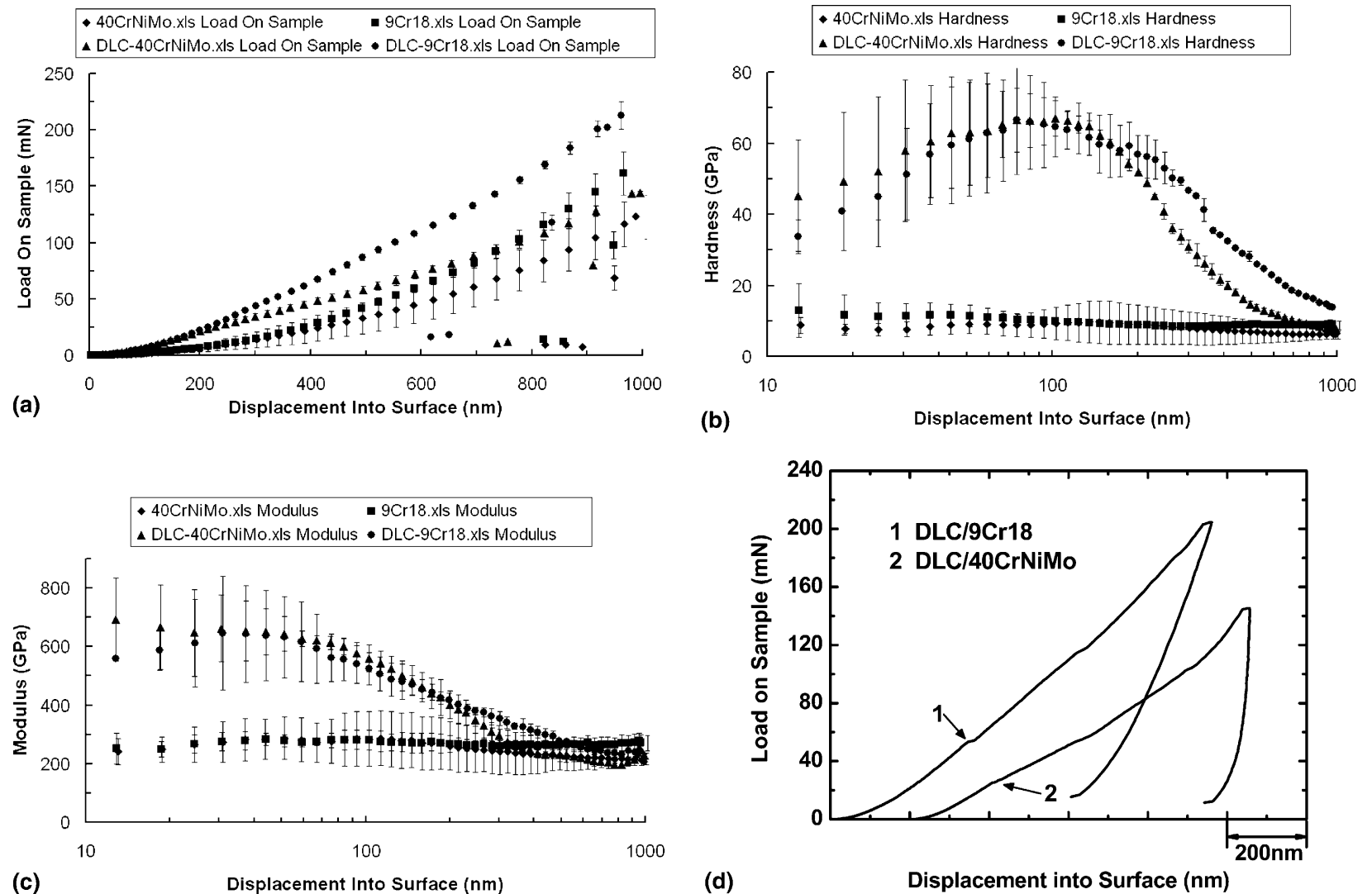

Fig. 1. The nanoindentation results with five separate indents for the four samples: 40CrNiMo, 9Cr18, DLC/40CrNiMo, and DLC/9Cr18. (a)-(c) show the load on sample, hardness, and modulus as a function of displacement into surface, respectively. (d) shows the typical load on sample as a function of displacement into surface of DLC/40CrNiMo and DLC/9Cr18.

represent the formation of subsurface cracks [7]. The load/depth values of the first distinct steps as indicated by arrows in Fig. 1(d) are $53 \mathrm{mN} / 350 \mathrm{~nm}$ for DLC/ 9Cr18 and $25 \mathrm{mN} / 210 \mathrm{~nm}$ for DLC/40CrNiMo. This shows that DLC/9Cr18 presents the higher load-carrying capacity and better adhesion behavior.

\subsection{Nanoscratch properties}

Fig. 2(a) shows the scratch depth as a function of the scratch distance or the normal load of organic/DLC/ $40 \mathrm{CrNiMo}$ during the pre-scan, scratching, and postscan processes. The pre-scan curve corresponds to the profile of the initial surface, the scratching curve corresponds to the tip penetration profile during testing, and finally the post-scan curve represents the residual depth after scratching, which can be used to assess the surface damage and any elastic/plastic deformation. A negative depth corresponds to the scratch tip being pushed into the sample, and a positive depth indicates outward blistering of the surface or the accumulation of debris in the scratch test [8].

The scratching curve in Fig. 2(a) was used to study the behavior of DLC coatings during the loading pro-
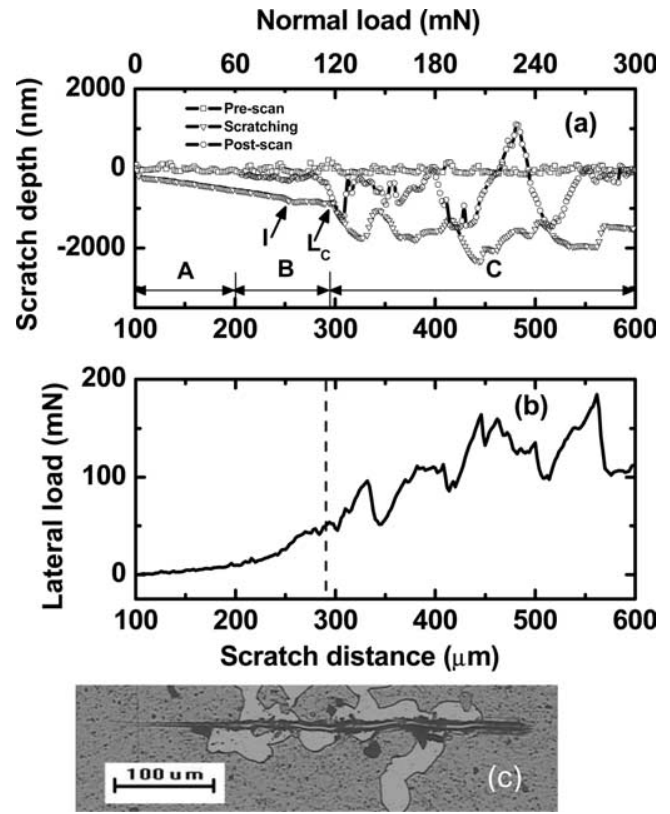

Fig. 2. The nanoscratch results with the ramping normal load of $300 \mathrm{mN}$ for organic/DLC/40CrNiMo: (a) the scratch depth as a function of the scratch distance and the normal load; (b) the lateral load as a function of the scratch distance and the normal load; (c) the optical image of scratch damage. 
cess. It is evident that the scratching depth linearly increases as a function of the ramping increase of the normal load from 0 to $84 \mathrm{mN}$ or scratch distance from 0 to $240 \mu \mathrm{m}$. The coefficient of friction also increases linearly from 0.15 to 0.40 (Fig.4(c)), which is attributed to scratch processes from organic to substrate. Then, the slope of scratching curve decreases from 240 to $290 \mu \mathrm{m}$, the coefficient of friction keeps a constant of 0.40 , which is the same as that of
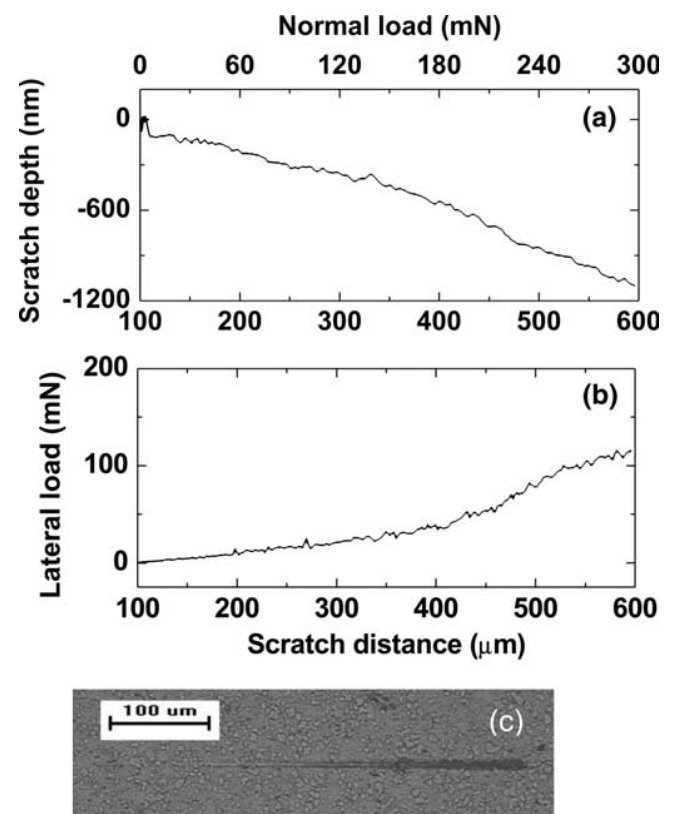

Fig. 3. The nanoscratch results with the ramping normal load of $300 \mathrm{mN}$ for organic/DLC/9Cr18: (a) the scratch depth as a function of the scratch distance and the normal load; (b) the lateral load as a function of the scratch distance and the normal load; (c) the optical image of scratch damage.
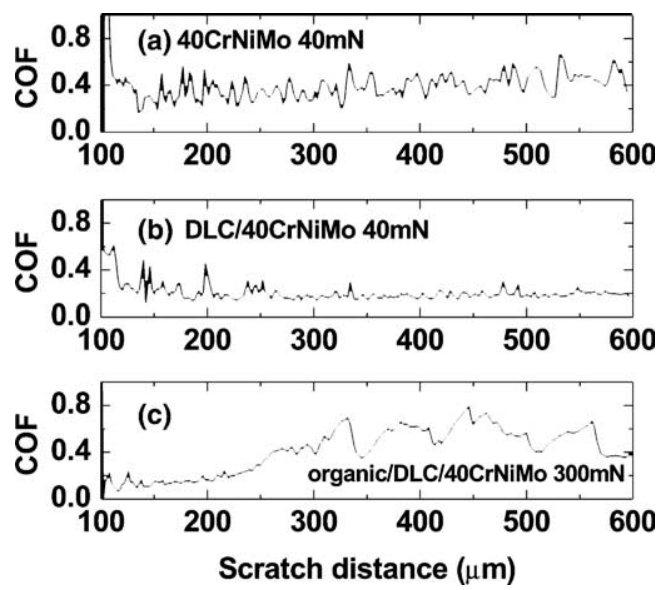

Fig. 4. Coefficient of friction as a function of the scratch distance under various maximum normal loads for different samples: (a) $40 \mathrm{CrNiMo}$ with $40 \mathrm{mN}$; (b) DLC/40CrNiMo with $40 \mathrm{mN}$; (c) organic/DLC/ 40CrNiMo with $300 \mathrm{mN}$.
40CrNiMo (Fig. 4(a)). It means that the tip has scratched into the substrate. Finally, there is an abrupt change in the scratching curve, implying that the coating peeled off during this scratch process and the coefficient of friction also suddenly changes. The loads associated with this event are termed as the critical loads $\left(\mathrm{L}_{\mathrm{C}}\right)$ [6], where the normal load is $114 \mathrm{mN}$ and the lateral load is $50 \mathrm{mN}$ (see Fig. 2(b)) for the organic/DLC/40CrNiMo. The post-scan curve nearly coincides with the pre-scan over the range A, although the profile of the scratch scan goes up from 100 to $200 \mu \mathrm{m}$. This indicates that the deformation of the DLC coatings almost fully recover under these loads, in which the initial elastic deformation is coating-dependent and is hardly related to the substrate. Further increasing scratch load, the pre-scan and post-scan curves gradually separated, which can be seen in the range B from 200 to $290 \mu \mathrm{m}$, where the plastic deformation happened and the residual depth gradually increased. Over the range $\mathrm{C}$, the post-scan curve abruptly changes. The optical image in Fig. 2(c) reveals that there was nearly no scratch trace over the range A. The profile is initially smooth and shallow, then gradually broadens and deepens with load increasing over the range $\mathrm{B}$. The large fluctuations in the post-scan curve can be found over the range $\mathrm{C}$, implying that the coatings have been delaminated, in which large amounts of coating have pulled off due to the high brittleness and intrinsic stress of the coatings [9]. It should be noted that, after unloading, the coatings were delaminated from the substrate due to the asynchronous recovery between the coating and the substrate in the range from 240 to $290 \mu \mathrm{m}$ (marked as $\mathrm{I}$ and $\mathrm{L}_{\mathrm{C}}$ ).

Fig. 3 shows the typical scratch results of organic/ DLC/9Cr18. It is evident in Figs. 3(a) and (b) that the scratch depth and the lateral load linearly increase as a function of the normal load from 0 to $180 \mathrm{mN}$ or scratch distance from 100 to $400 \mu \mathrm{m}$. The coefficient of friction also increases linearly from 0.15 to 0.20 in Fig. 5(c). It is shown that the tip scratched into the organic/DLC. Then, the slopes of the scratch depth curve and the lateral load curve increase from 400 to $600 \mu \mathrm{m}$, the coefficient of friction increases from 0.20 to 0.40 and keeps a constant of 0.40 , which is the same as that of $9 \mathrm{Cr} 18$ (Fig. 5(a)). It is indicated that the tip scratched into the substrate $9 \mathrm{Cr} 18$. Fig. 3(c) shows that the scratch response was controlled by elastic-plastic deformation, no delamination.

Figs. 4(a) and 5 (a) give the friction coefficients of 0.45 and 0.40 for $40 \mathrm{CrNiMo}$ and $9 \mathrm{Cr} 18$ substrates, respectively. Figs. 4(b) and 5 (b) give the friction coefficient of 0.20 for both DLC/40CrNiMo and DLC/ 40CrNiMo. Therefore, the coefficients of friction for 40CrNiMo, 9Cr18, DLC, and organic coating are approximately $0.45,0.40,0.20$ and 0.15 , respectively. It 

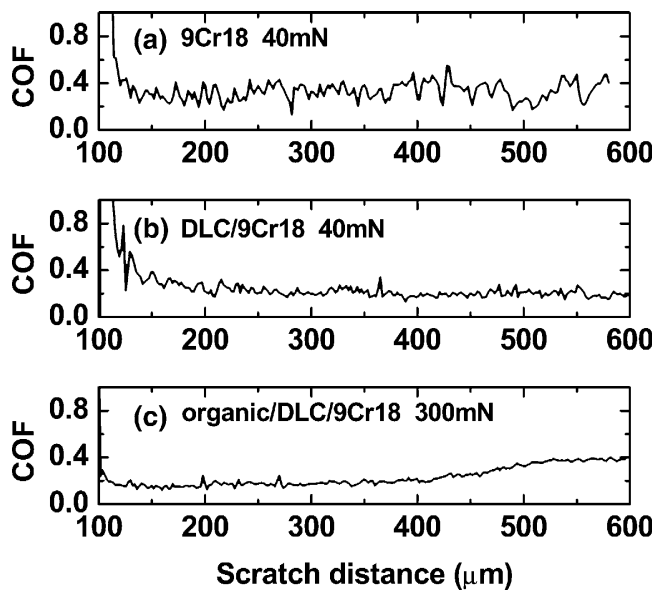

Fig. 5. Coefficient of friction as a function of the scratch distance under various maximum normal loads for different samples: (a) 9Cr18 with $40 \mathrm{mN}$; (b) DLC/9Cr18 with $40 \mathrm{mN}$; (c) organic/DLC/9Cr18 with $300 \mathrm{mN}$.

is indicated that the lubricant effect of DLC and the organic coating are remarkable.

\section{Conclusion}

In this study, we studied the nanomechanical and nanotribological properties of the solid lubricative DLC coatings on two different substrates. Here, are the main conclusions:

1. Compared with the steel substrates, the mechanical and wear resistance properties of DLC/steels are significantly improved. The lubricative effect of DLC coatings and the organic coatings is remarkable.

2. $9 \mathrm{Cr} 18$ steel is a better substrate. On the one hand, the load-carrying capacities of $9 \mathrm{Cr} 18$ and DLC/9Cr18 are higher than those of $40 \mathrm{CrNiMo}$ and $\mathrm{DLC} /$ $40 \mathrm{CrNiMo}$. On the other hand, the delamination of DLC/40CrNiMo is more severe. This may be attributed to the better interface properties between DLC and $9 \mathrm{Cr} 18$ steel substrates.
3. The nanoindentation/scratch technique can provide us very useful information about the near-surface elastic-plastic deformation, fracture, friction and wear properties.

\section{Acknowledgments}

The authors thank Mrs. Xiulan Wang in Beijing Research Institute of Aerospace Materials and Technology for the preparation of DLC coatings and Dr. Cunyi Xie in MTS Systems (China) Inc., for helpful discussion. This project was supported by the National Natural Science Foundation of China (10372103 and 10432050) and Chinese Academy of Sciences (KJCX2-SW-L2).

\section{References}

[1] Lifshitz Y. Diamond-like carbon - present status. Diam Relat Mater 1998;8(8-9):1659-76.

[2] Staedler T, Schiffmann K. Correlation of nanomechanical and nanotribological behavior of thin DLC coatings on different substrates. Surf Sci 2001;482-485:1125-9.

[3] Oliver WC, Pharr GM. An improved technique for determining hardness and elastic modulus using load and displacement sensing indentation experiments. J Mater Res 1992;7(6):1564-83.

[4] Lee KW, Chung YW, Chan CY, Bello I, et al. An international round-robin experiment to evaluate the consistency of nanoindentation hardness measurements of thin films. Surf Coat Tech 2003;168:57-61.

[5] Moody NR, Strojny A, Medlin DL, Talin A, Gerberich AA. Substrate composition effects on the interfacial fracture of tantalum nitride films. J Mater Res 1999;14(6):2306-13.

[6] Bhushan B. Handbook of micro/nanotribology. 2nd ed. Boca Raton: CRC Press; 1999.

[7] Bhushan B, Li X. Nanomechanical characterization of solid surfaces and thin films. Int Mater Rev 2003;48(3):125-64.

[8] Huang LY, Zhao JW, Xu KW, Lu J. A new method for evaluating the scratch resistance of diamond-like carbon films by the nanoscratch technique. Diam Relat Mater 2002;11:1454-9.

[9] Huang LY, Zhao JW, Xu KW, Lu J. Analysis of nano-scratch behavior of diamond-like carbon films. Surf Coat Tech 2002;154:232-6. 